(1)

CrossMark

\title{
The (in)significance of TB and COVID-19 co-infection
}

To the Editor:

We read with great interest the research letter by TADOLINI et al. [1], in which they have published the first cohort of 49 cases of tuberculosis (TB) and coronavirus disease 20019 (COVID-19) co-infection. However, a few issues regarding the letter need to be addressed.

The authors categorised the patients with TB and COVID-19 co-infection into 3 groups based on timing of their diagnosis. However, in view of the difference in the natural history of TB (chronic course) and COVID-19 (acute), categorising 14 patients as having COVID-19 prior to TB (median time interval of 4 days between the two diagnosis) and nine as diagnosed simultaneously (within the same week) seems inappropriate. Since TB has an insidious onset, it is obvious that TB was present before COVID-19 infection in both the subgroups, although the diagnosis was made at different times. In fact, it may be right to say that all the three subgroups actually constitute a single group of old/active TB patients who developed COVID-19 infection. COVID-19 has probably just unmasked some of the subtle active TB cases that were responsible for hidden transmission in the general population [2]. Superimposed COVID-19 has brought them to the hospital to get a timely diagnosis.

Considering the high worldwide prevalence of TB and increasing burden of COVID-19, the co-infection seems more likely to be a co-incidental occurrence rather than a causal association. It is likely that patients with active TB will have more time to get exposed to COVID-19 infection due to chronic course of TB. The hypothesis is also supported by a higher percentage of multidrug-resistant TB patients in the present study, as these patients are on a prolonged treatment and harbour TB disease for a longer period of time. However, a well-designed prospective cohort study is required to prove any causal association between the two diseases, as also concluded by the authors.

Apart from assessing association, the other main concern about this co-infection is the mortality associated with it. The present study showed a mortality of $12.3 \%$ in the patients with dual infection, which is much higher than isolated COVID-19 [3]. However, this apparent higher mortality cannot be attributed to the dual infection from this cohort as the majority of patients with fatal disease had proven risk factors for mortality ( $>60$ years age and one or more comorbidity) that might have distorted the figures.

Irrespective of the probable temporal association between COVID-19 and TB, both the infectious diseases may have synergistic impact on social and economic impact worldwide. This is because both the diseases are expected to spread in overcrowded areas with poor and undernourished populations [4]. High TB burden countries have a huge number of patients with post TB lung sequele and the outcome of COVID-19 in such patients is unknown so far. It is important to understand from this analysis that in the fight against COVID-19 pandemic, we should not forget to suspect and manage TB appropriately, which is still one of the leading infectious causes of death worldwide.

@ERSpublications

In the fight against COVID-19 pandemic, we should not forget to suspect and manage TB appropriately, as it is still one of the leading infectious causes of death worldwide https://bit.ly/ 30FZs9C

Cite this article as: Khurana AK, Aggarwal D. The (in)significance of TB and COVID-19 co-infection. Eur Respir J 2020; 56: 2002105 [https://doi.org/10.1183/13993003.02105-2020].

Alkesh Kumar Khurana ${ }^{1}$ and Deepak Aggarwal ${ }^{2}$

${ }^{1}$ Pulmonary Medicine, All India Institute of Medical Sciences, Bhopal, India. ${ }^{2}$ Pulmonary Medicine, Govt Medical College and Hospital, Chandigarh, India.

Correspondence: Alkesh Kumar Khurana, Pulmonary Medicine, All India Institute of Medical Sciences, Bhopal, Madhya Pradesh, India. E-mail: lungcancer@rediffmail.com 
Received: 1 June 2020 | Accepted: 8 June 2020

Conflict of interest: A.K. Khurana has nothing to disclose. D. Aggarwal has nothing to disclose.

\section{References}

1 Tadolini M, Codecasa LR, Garcia-Garcia J-M, et al. Active tuberculosis, sequele and COVID-19 co-infection:first cohort of 49 cases. Eur Respir J 2020; 56: 2001398.

2 Saunders MJ, Evans CA. COVID-19, tuberculosis and poverty: preventing a perfect storm. Eur Respir J 2020; 56: 2001348.

3 Guan WJ, Ni ZY, Hu Y, et al. Clinical characteristics of coronavirus disease 2019 in China. N Engl J Med 2020; 382: $1708-1720$.

4 Wingfield T, Tovar MA, Datta S, et al. Addressing social determinants to end tuberculosis. Lancet 2018; 391: $1129-1132$.

\section{On tuberculosis and COVID-19 co-infection}

From the authors:

We wish to thank A.K. Khurana and D. Aggarwal for their interest in our research letter and comments.

In their correspondence, the authors raised two important issues, namely the possible association between tuberculosis (TB) and coronavirus disease 2019 (COVID-19) (can infection by severe acute respiratory syndrome coronavirus 2 (SARS-CoV-2) re-activate TB?), and the effects of TB on early mortality in co-infected patients.

Our research letter reported the first cohort of patients with diagnosis of TB (including post-treatment sequelae) and COVID-19. The article was aimed at reporting what was observed at the beginning of the epidemic among some of the most affected countries. This explains the small numbers described and the countries involved. At the time the article was submitted, several countries in Africa, Europe and Latin America represented in the Global Tuberculosis Network (GTN) had no TB/COVID-19 patients to report.

In the absence of previous cohorts and scientific information on TB/COVID-19 co-infection, we have described the timing of diagnosis of the two diseases, observing that one third had COVID-19 diagnosed prior to TB and $18 \%$ were diagnosed simultaneously.

We agree, it is possible that the diagnosis of COVID-19 was made before TB because of acute onset of symptoms caused by SARS-CoV-2 in addition to the alarm generated by the COVID-19 pandemic, which determined rapid access to radiological examinations and subsequent discovery of underlying TB. In fact we commented on this in point 3 of our article [1], and we abstained from making any clear statement about causal association. However, we could not exclude that the infection by SARS-CoV-2 or the drugs utilised might have accelerated the progression of a pre-existing TB infection to disease.

However, apart from the speculation on what disease comes first, it is evident that the co-existence of TB and COVID-19 poses a challenge in differential diagnosis [1].

The study was observational, and based on a relatively small cohort, and therefore we fully agree that larger prospective studies are necessary to shed further light on this to establish whether there is an association or not.

@ERSpublications

COVID-19 may boost tuberculosis given infection and mortality, further studies are needed https://bit. ly/2Z2r5XO

Cite this article as: Tadolini M, García-García J-M, Blanc F-X, et al. On tuberculosis and COVID-19 co-infection. Eur Respir J 2020; 56: 2002328 [https://doi.org/10.1183/13993003.02328-2020]. 
A.K. Khurana and D. Aggarwal also raised the important question of whether TB has a real effect or "weight" in increasing the probability of death in COVID-19 patients.

The issue has been described in a second article [2] which reports the findings of 69 patients from our original cohort plus a second cohort [3] which was managed in a reference hospital in Northern Italy.

The patients more likely to die were those of older age with pre-existing comorbidities [2].

It is important to emphasise that the cohort of young migrants without comorbidities reported elsewhere $[2,3]$ experienced a milder form of COVID-19 with no deaths.

However, in countries where risk factors for mortality are highly prevalent among young individuals (smoking, alcohol and substance abuse, HIV co-infection, among others), particularly in the presence of drug resistance and difficult access to diagnosis (delayed diagnosis), the impact of mortality may be higher. We agree that, in resource-limited settings, poverty and malnutrition might play an important role in increasing morbidity and mortality.

Furthermore, we do agree that the population of individuals with post-TB treatment sequelae deserves further evaluation, given the potential effect of both TB and COVID-19 on quality of life and subsequent need for rehabilitation [4-6].

In order to better understand the implication of TB and COVID-19 co-infection the study is continuing: more countries and a larger sample size will help answering some of the questions left open by our original study [1]. We will be happy to collaborate with all interested colleagues.

Marina Tadolini ${ }^{1}$, José-María García-García $\oplus^{2}$, François-Xavier Blanc $\oplus^{3}$, Sergey Borisov ${ }^{4}$, Delia Goletti ${ }^{5}$, Ilaria Motta ${ }^{6}$, Luigi Ruffo Codecasa ${ }^{7}$, Simon Tiberi ${ }^{8,9}{ }^{8,}$, Giovanni Sotgiu ${ }^{10}$ and Giovanni Battista Migliori ${ }^{11}$, on behalf of the GTN TB/COVID group ${ }^{12}$

${ }^{1}$ Unit of Infectious Diseases, Dept of Medical and Surgical Sciences, Alma Mater Studiorum University of Bologna, Bologna, Italy. ${ }^{2}$ Tuberculosis Research Programme (PII-TB), SEPAR, Barcelona, Spain. ${ }^{3}$ Centre Hospitalier Universitaire, Nantes, France. ${ }^{4}$ Moscow Research and Clinical Center for TB Control, Moscow, Russian Federation. ${ }^{5}$ Translational Research Unit, National Institute for Infectious Diseases "L. Spallanzani”, IRCCS, Rome, Italy. ${ }^{6}$ Dipartimento di Scienze Mediche, Clinica Universitaria Malattie Infettive, Ospedale Amedeo di Savoia, Torino, Italia. ${ }^{7} \mathrm{~TB}$ Reference Centre, Villa Marelli Institute, Niguarda Hospital, Milan, Italy. ${ }^{8}$ Blizard Institute, Barts and The London School of Medicine and Dentistry, Queen Mary University of London, London, UK. ${ }^{9}$ Division of Infection, Royal London Hospital, Barts Health NHS Trust, London, UK. ${ }^{10}$ Clinical Epidemiology and Medical Statistics Unit, Department of Medical, Surgical and Experimental Sciences, University of Sassari, Sassari, Italy. ${ }^{11}$ Servizio di Epidemiologia Clinica delle Malattie Respiratorie, Istituti Clinici Scientifici Maugeri IRCCS, Tradate, Italy. ${ }^{12} \mathrm{~A}$ list of members of the GTN TB/COVID group can be found in the acknowledgements section.

Correspondence: Giovanni Battista Migliori, Servizio di Epidemiologia Clinica delle Malattie Respiratorie, Istituti Clinici Scientifici Maugeri IRCCS, Via Roncaccio 16, Tradate, Varese, 21049, Italy. E-mail: giovannibattista.migliori@icsmaugeri.it

Received: 15 June 2020 | Accepted: 15 June 2020

Acknowledgements: The article is part of the scientific activities of the Global Tuberculosis Network (GTN); GREPI (Groupe de Recherche et d'Enseignement en Pneumo-Infectiologie), a working group from SPLF (Société de Pneumologie de Langue Française); SEPAR (Sociedad Española de Neumología and Cirugía Torácica); Moscow Society of Phtisiology and of the WHO Collaborating Centre for Tuberculosis and Lung Diseases, Tradate, ITA-80, 2017-2020- GBM/RC/LDA).

The members of the GTN TB/COVID group are: Jan-Willem Alffenaar (Australia); Pierre Bachez (Belgium); Denise Rossato Silva (Brazil); Claire Andréjak, François-Xavier Blanc, Samir Dourmane, Mathilde Fréchet Jachym, Antoine Froissart, Soazic Grard, Armine Izadifar, Damien Le Du, Frédéric Rivière and Frédéric Schlemmer (France); Rosella Centis, Luigi Ruffo Codecasa, Lia D’Ambrosio, Vania Giacomet, Delia Goletti, Gina Gualano, Filippo Lipani, Giovanni Battista Migliori, Ilaria Motta, Maria Musso, Fabrizio Palmieri, Emanuele Pontali, Matteo Saporiti, Paolo Scarpellini, Giovanni Sotgiu, Antonio Spanevello, Claudia Stochino, Marina Tadolini, Alessandro Torre, Simone Villa and Dina Visca (Italy); Evgeny Belilovski, Sergey Borisov and Elena Sumarokova (Russian Federation); Paul Anantharajah Tambyah and Catherine Wei Min Ong (Singapore); José Cardoso-Landivar, María-Luiza De Souza-Galvão, Angel Dominguez-Castellano, José-María García-García, Margarita Marín Royo, Teresa Rodrigo, Adrián Sánchez-Montalvá, Eva Tabernero and Miguel Zabaleta Murguiondo (Spain); Pierre-Alexandre Bart and Jesica Mazza-Stalder (Switzerland); Simon Tiberi (UK).

Conflict of interest: None declared.

\section{References}

1 Tadolini M, Codecasa LR, García-García JM, et al. Active tuberculosis, sequelae and COVID-19 co-infection: first cohort of 49 cases. Eur Respir J 2020; 56: 2001398.

2 Motta I, Centis R, D'Ambrosio L, et al. Tuberculosis, COVID-19 and migrants: preliminary analysis of deaths occurring in 69 patients from two cohorts. Pulmonology 2020; 26: 233-240.

3 Stochino C, Villa S, Zucchi P, et al. Clinical characteristics of COVID-19 and active tuberculosis co-infection in an Italian reference hospital. Eur Respir J 2020; in press [https://doi.org/10.1183/13993003.01708-2020]. 
4 Visca D, Zampogna E, Sotgiu G, et al. Pulmonary rehabilitation is effective in patients with tuberculosis pulmonary sequelae. Eur Respir J 2019; 53: 1802184

5 Zumla A, Marais BJ, McHugh TD, et al. The increasing threat of respiratory tract infections to global health security - COVID-19 and tuberculosis. Int J Tuberc Lung Dis 2020 [https://doi.org/10.5588/ijtld.20.0387].

6 Visca D, Tiberi S, Pontali E, et al. Tuberculosis in the time of COVID-19: quality of life and digital innovation. Eur Respir J 2020; 56: 2001998.

Copyright (CERS 2020.

This version is distributed under the terms of the Creative Commons Attribution Non-Commercial Licence 4.0. 